\title{
Metastatic breast cancer in patients with schizophrenia
}

\author{
AARON A. MEYER ${ }^{1,2}$, M. HWANG ${ }^{1,2}$, M. FARASATPOUR ${ }^{1,2}$, R. JANARDHAN ${ }^{1,2}$, \\ J.A. MARGENTHALER ${ }^{3}$, K.S. VIRGO ${ }^{4}$ and FRANK E. JOHNSON ${ }^{1,2}$ \\ ${ }^{1}$ Saint Louis University; ${ }^{2}$ St. Louis VA Medical Center; ${ }^{3}$ Washington University, St. Louis, MO; \\ ${ }^{4}$ American Cancer Society, Atlanta, GA, USA
}

Received July 11, 2012; Accepted October 30, 2012

DOI: $10.3892 / \mathrm{mco} .2012 .44$

\begin{abstract}
Breast cancer is a major health problem worldwide. The median survival duration for patients with metastatic breast cancer is two to three years. Approximately $1 \%$ of populations worldwide have schizophrenia. The manner in which schizophrenic patients fare when diagnosed with metastatic breast carcinoma (MBC) was evaluated. We queried the National Department of Veterans Affairs (DVA) datasets using computer codes for a pre-existing diagnosis of schizophrenia and a later diagnosis of breast carcinoma. Chart-based data concerning the identified subjects were then requested. Previously determined inclusion and exclusion criteria were applied to select evaluable patients from the medical records, prior to extracting demographic details and data concerning the treatment course in each subject. Ten patients had distant metastases at initial diagnosis, while seven developed MBC following prior curative-intent treatment. Two patients refused therapy. Ten did not comply with recommended management. Five harmed or threatened physicians, other caregivers or themselves. Schizophrenic patients with MBC often fail to understand the nature of their illnesses. Often they do not accept palliative treatment, while a number of them do not comply with therapy, once initiated. They often exhibit behaviors that are detrimental to themselves or others. Formal psychiatric consultation is therefore necessary in patients. Several detrimental behaviors may be predicted reliably by history alone.
\end{abstract}

\section{Introduction}

Breast carcinoma is a major health problem worldwide. In the USA, it is the most common cancer in women, with the exception of non-melanoma skin cancer (1). Approximately $20 \%$ of women with early breast cancer develop distant metastases. Approximately $10 \%$ of patients have distant metastases at diagnosis in the USA. The median survival duration for

Correspondence to: Dr Frank E. Johnson, Department of Surgery, P.O. Box 15250, Saint Louis University Medical Center, 3635 Vista Ave., St. Louis, MO 63110-0250, USA

E-mail: frank.johnson1@va.gov

Key words: breast cancer, metastatic disease, schizophrenia such patients is two to three years and there are several treatment options available (2). With current treatment, disabling symptoms are likely to be delayed for months or years. Breast cancer comprises $<1 \%$ of cancers in men. Less than $1 \%$ of the breast cancers in the US occur in males (3). In a study from the Department of Veterans Affairs (DVA), the median survival duration for females with metastatic breast cancer (MBC) was greater compared to males (4).

Approximately $1 \%$ of populations worldwide have schizophrenia. The disease is considered to be multifactorial. It is strongly associated with a family history of schizophrenia, with an incidence of $6.5 \%$ in first-degree relatives of affected subjects and $40 \%$ in both monozygotic twins born to a couple with one affected parent (5). Several other risk factors have been shown to be correlated with schizophrenia, including advanced paternal age (6). Increased numbers of specific genetic variants, notably the 22q11 deletion, are associated with a notable increase in incidence (7). Since the underlying mechanism is thought to involve abnormalities in neural circuitry, circuit analysis is one of the numerous approaches used to understand this complex disease. Abnormal neural connections between the dorsolateral prefrontal cortex and hippocampal formation are known to be associated with schizophrenia (8).

The lifespan of affected patients is $\sim 25$ years shorter compared to people who do not have a major mental disorder (9). Schizophrenic patients often have difficulty with daily functioning and social interaction, while the disease is a major financial burden to society (10). Various abnormal behaviors present difficulties in clinical management. Individuals with schizophrenia are four to six times more likely to exhibit violent behaviors compared to the general population (11). Their suicide rate is $5-13 \%$ higher compared to people without this illness. Approximately 40-60\% of affected patients abuse drugs and homelessness is very common (12). Approximately $75 \%$ of patients do not adhere to their antipsychotic medication regimen in a two-year period following discharge from inpatient facilities, and their rate of non-adherence to antipsychotic agents is almost identical to their rate of non-adherence to other medications (13).

Data concerning the rate of adherence to recommended cytotoxic chemotherapy and endocrine therapy regimens in patients not selected for schizophrenia is scanty. In their study, Hershman et al (14) have noted a 7-10\% rate of discontinuation of adjuvant endocrine therapy in patients with early-stage 
breast cancer in this population. Notably, Sharma et al (15) reported no statistically significant differences in the treatment course in patients with schizophrenia and those without schizophrenia. However, Tran et al (16) indicated that the risk of death from breast cancer in such patients is significantly higher compared to the general population. In this study, we evaluated the manner in which schizophrenic patients fare in a large, non-referral-based population when diagnosed with MBC.

\section{Materials and methods}

Study approval. Institutional Review Boards (IRB) approval was obtained for this study. The Patient Treatment File (PTF) database and the national archiving system for inpatient data in the DVA medical system were accessed to retrieve data of interest. Security clearance was obtained to use the DVA Data Processing Center in Austin, TX, USA, which has data from all the administrative databases of DVA facilities. Its computer components include the IBM Z900 (2064-2CS) and IBM Z890 (2086-320)\Sun E10000 HD-UX mainframes coupled with EMC storage computers (EMC 8730 and 8830; IBM, Armonk, NY, USA). Information on the PTF database may be retrieved from the Veterans Information Resource Center (VIREC) website at http://www.virec.research.va.gov.

Database search. National DVA datasets using computer codes were queried for a pre-existing diagnosis of schizophrenia and a later diagnosis of breast carcinoma. The PTF database was searched to identify patients with the International Classification of Diseases, Ninth Revision, Clinical Modifications (ICD-9-CM) (www.cdc.gov/nchs/icd/ icd9.htm) diagnostic codes for schizophrenia or schizoaffective disorder (codes 295.00-295.85) and subsequently diagnosed with breast cancer (codes 174-174.9, 198.2, 198.81, $217,233.0,611.72$ ) during the fiscal years 1999-2005. The PTF database identified appropriate patients based on these computer codes and yielded demographic information as well as data on comorbidities.

Patient data. For additional data, chart-based information was requested from the DVA Medical Centers, where patients had received their breast carcinoma care. These data included discharge summaries, progress notes, operative reports, medical oncology consultation summaries, psychiatric consultation notes and pathology reports. To obtain further information regarding the psychiatric history of each patient, medical records were requested predating the admission for breast cancer. From this pool of patients, we selected patients diagnosed with MBC were selected. The patients were then grouped into those initially presenting with $\mathrm{MBC}(\mathrm{n}=10)$ and those with MBC after prior curative-intent treatment $(n=7)$.

Descriptive statistics were utilized to analyze the demographic information, clinical course and outcomes of the patients. Patients were excluded from further review according to pre-specified criteria: medical chart data not available to provide adequate information; apparent coding errors that, upon chart review, demonstrated no evidence of schizophrenia or schizoaffective disorder; coding errors that demonstrated no evidence of a breast carcinoma; diagnosis of breast cancer prior to diagnosis of schizophrenia and patients assessed by their physicians as inappropriate candidates for adjuvant endocrine and/or cytotoxic chemotherapy, for any reason. We assumed a variable of interest was present when mentioned as present in the medical record. We assumed it was absent when not mentioned in the record. We recognized that this would lead to a conservative estimate of the presence of most variables.

\section{Results}

Patient data. Approximately 25 million veterans are eligible for VA healthcare, while $\sim 5$ million veterans receive care in DVA medical centers each year (Congressional Budget Office) (17). During the time period of our study, 10 patients were identified with an initial diagnosis of $\mathrm{MBC}$ as well as seven patients with MBC after initial curative-intent treatment.

There were 10 patients with no prior diagnosis of (or treatment for) breast carcinoma, who presented initially with MBC. Of these 10 patients, two lived alone, five with a family member, while one was homeless. Data on the remaining two were not available. Five patients were documented not to have a family history of breast cancer. Additional data is summarized in Table I.

Evidence of thought disorder and abnormal behavior in the 10 patients. Four of the 10 patients were diagnosed with paranoid, one with catatonic and two with undifferentiated schizophrenia, while three had schizoaffective disorder. Based on the diagnostic criteria for schizophrenia (Table IV), seven patients had a concurrent history of other Axis I psychiatric disorders, such as depression, bipolar disorder and post-traumatic stress disorder (PTSD) (18). One of the seven patients had major depression, one had PTSD, two had bipolar disorder, two had a psychotic disorder-not otherwise specified (NOS) and one had a psychotic disorder-NOS with PTSD.

Affect is often abnormal in schizophrenic patients. The affect of two patients was described as full-range and appropriate, of one patient as constricted, one blunted/appropriate, while two patients affect was described as blunted/guarded. One patient had affect described as dull and one patient as labile. Delusions are very common in schizophrenic patients. Of the 10 patients, one had delusions of grandiosity, two had persecutory delusions and one had thought-insertion delusions. Two patients had a history of disruptive behavior, one of whom set fire to her own clothes after being denied her holistic medicines. Additional evidence of abnormal thoughts and behaviors are shown in Tables II and III.

Three of the 10 patients were taking phenothiazines (haloperidol, perphenazine and trifluoperazine). Seven patients were taking atypical antipsychotics (most commonly loxapine, olanzapine and risperidone), with six of the seven experiencing adverse reactions to their antipsychotic medication.

Symptoms and treatment. Seven of the 10 patients inappropriately delayed seeking medical attention for the MBC. For four of the seven, the mean time from onset of MBC symptoms to initial treatment was 259 days and two of these seven waited $>1$ year. The mean waiting time from symptom onset to palliative surgery was three years, with two of the seven waiting $>4$ years. Three of the 10 patients could not give a coherent 
Table I. Demographic data documented in DVA records.

\begin{tabular}{|c|c|c|}
\hline Variable & $\begin{array}{l}\text { MBC at initial diagnosis of cancer } \\
\qquad(\mathrm{n}=10)\end{array}$ & $\begin{array}{l}\text { MBC after initial curative-intent treatment } \\
\qquad(\mathrm{n}=7)\end{array}$ \\
\hline Gender (male:female) & $1: 9$ & $3: 4$ \\
\hline Ethnicity & $5 \mathrm{C}: 4 \mathrm{~A}: 1 \mathrm{U}$ & $3 \mathrm{C}: 2 \mathrm{~A}: 1 \mathrm{H}: 1 \mathrm{U}$ \\
\hline Mean age (years) & 54 & 54 \\
\hline Range & $40-81$ & $43-70$ \\
\hline \multicolumn{3}{|l|}{ Comorbid conditions } \\
\hline $\mathrm{HTN}$ & 3 & 5 \\
\hline DM & 2 & 2 \\
\hline Obesity & 4 & 6 \\
\hline COPD & 2 & 1 \\
\hline Cronic renal failure & 1 & 0 \\
\hline CAD or prior $\mathrm{MI}$ & 0 & 3 \\
\hline \multicolumn{3}{|l|}{ Breast cancer histology } \\
\hline Ductal adenocarcinoma & 6 & 5 \\
\hline Lobular carcinoma & 1 & 0 \\
\hline Inflammatory breast cancer & 2 & 0 \\
\hline Atypical medullary carcinoma & 0 & 1 \\
\hline Unspecified breast carcinoma & 1 & 1 \\
\hline Current illicit drug abuse & 2 & 2 \\
\hline Current alcohol abuse & 4 & 5 \\
\hline
\end{tabular}

DVA, Department of Veterans Affairs; MBC, metastatic breast cancer; C, Caucasian; A, African-American; U, unknown; H, Hispanic; HTN hypertension; DM, diabetes mellitus; COPD, chronic obstructive pulmonary disease; CAD, coronary artery disease; MI, myocardial infarction.

Table II. Evidence of thought disorder noted in DVA records.

\begin{tabular}{|c|c|c|}
\hline Characteristic & $\mathrm{MBC}$ at initial diagnosis $(\mathrm{n}=10)$ & MBC after initial curative-intent treatment $(n=7)$ \\
\hline Confusion & 4 & 2 \\
\hline Lack of insight & 3 & 4 \\
\hline Hallucinations & 7 & 4 \\
\hline Delusions & 4 & 3 \\
\hline Paranoia & 8 & 5 \\
\hline Abnormal affect & 6 & 3 \\
\hline Ideas of reference ${ }^{a}$ & 5 & 2 \\
\hline Thought blocking ${ }^{\mathrm{b}}$ & 1 & 1 \\
\hline Disorganized thought process & 4 & 4 \\
\hline Suicidal ideation & 2 & 2 \\
\hline Homicidal ideation & 2 & 0 \\
\hline
\end{tabular}

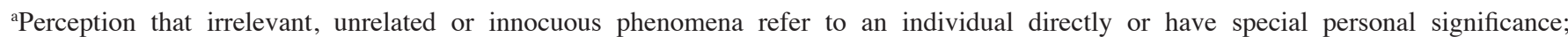
bself-interruption of speech before completion; DVA, Department of Veterans Affairs; MBC, metastatic breast cancer.

history. One patient believed that someone had put an egg into her right breast and a radio transmitter into her left one. Three patients were non-compliant with their antipsychotic medica- tion regimen when offered medications for $\mathrm{MBC}$. Of these three patients, none were compliant with the chemotherapy regimen. 
Table III. Evidence of abnormal behavior documented in DVA records.

\begin{tabular}{|c|c|c|}
\hline Variable & $\begin{array}{l}\text { MBC at initial diagnosis } \\
\qquad(\mathrm{n}=10)\end{array}$ & $\begin{array}{l}\text { MBC after initial curative-intent treatment } \\
\qquad(\mathrm{n}=7)\end{array}$ \\
\hline History of injuring caregivers & 3 & 2 \\
\hline History of verbal abuse of caregivers & 1 & 1 \\
\hline History of suicide attempt(s) & 1 & 1 \\
\hline $\begin{array}{l}\text { Inappropriate delay in seeking } \\
\text { medical attention for } \mathrm{MBC}\end{array}$ & 7 & 4 \\
\hline $\begin{array}{l}\text { History of non-compliance with antipsychotic } \\
\text { medication regimen prior to diagnosis of MBC }\end{array}$ & 7 & 3 \\
\hline Denial of breast cancer diagnosis & 3 & 0 \\
\hline Refusal of palliative treatment & 4 & 1 \\
\hline $\begin{array}{l}\text { Non-compliant with antipsychotic } \\
\text { medication regimen at the time } \\
\text { of diagnosis of } \mathrm{MBC}\end{array}$ & 3 & 0 \\
\hline $\begin{array}{l}\text { Compliant with antipsychotic medication } \\
\text { regimen at the time of diagnosis of MBC }\end{array}$ & 7 & 7 \\
\hline $\begin{array}{l}\text { Presentation with schizophrenic behavior(s) } \\
\text { typical of schizophrenic subjects }\end{array}$ & 4 & 1 \\
\hline Compliant with medication regimens & 1 & 1 \\
\hline Non-compliant with medication regimens & 3 & 0 \\
\hline
\end{tabular}

DVA, Department of Veterans Affairs; MBC, metastatic breast cancer.

Table IV. Diagnostic criteria for schizophrenia.

Categories Details/explanations

Characteristic symptoms

Social/occupational dysfunction

Duration

Organic etiology exclusion

Schizoaffective and mood disorder exclusion Pervasive developmental disorder relationship

Of the 10 patients in this group, seven underwent a computed tomography (CT), six a bone scan, three a chest ultrasound examination, six a chest radiograph and seven a mammogram during the extent-of-disease studies.

Seven of the 10 patients were offered cytotoxic chemotherapy, six received the initial doses of chemotherapy, although only two of the six completed $\geq 3$ cycles. One patient
Two or more symptoms during a one-month period: delusion, hallucinations, disorganized speech, disorganized/catatonic behavior and negative symptoms Marked decrease since onset of disturbance in: work, relationships and self-care Continuous signs $>6$ months

Includes: substance abuse or general medical conditions (brain lesions, seizures and endocrinopathies)

Episodes of major depression, manic or mixed episodes

Autism or other pervasive developmental disorders with characteristic symptoms of schizophrenia for $>1$ month is sufficient for the diagnosis of schizophrenia

All criteria for schizophrenia are taken from the Diagnostic and Statistical Manual of Mental Disorders, 4th edition (DSM-IV-TR $\left.{ }^{\circledR}\right)(18)$.

refused treatment saying 'God will help me through this'. Five of the 10 patients $(50 \%)$ were offered endocrine therapy, two refused therapy and three were compliant. Five of the 10 patients $(50 \%)$ were offered radiation therapy, two refused it and three complied with treatment. One patient's guardian refused to permit radiation treatment because she believed that the patient would not comply. The same patient drank sassa- 
fras tea as 'alternative treatment' and covered her fungating breast mass with a bandage. Seven of the 10 patients were offered palliative breast surgery, two of whom refused and five underwent the surgery. One patient who refused to take any psychiatric medication because hospital staff denied her shark cartilage for the breast cancer. Another patient refused the recommended treatment and took only herbal teas and vitamins as treatment.

Evidence of thought disorder and abnormal behavior in the seven patients. There were seven patients with prior curative-intent treatment, who presented with MBC. Of the seven patients, four lived alone and two were in a nursing home. One of the seven was institutionalized. Three of the seven patients had no documented family history of breast cancer. Additional information is summarized in Table I.

Two of these seven were diagnosed with paranoid schizophrenia, one had residual schizophrenia, three had schizoaffective disorder and one was unspecified. Four of the seven had Axis I personality disorders (three had major depression and one had bipolar disorder with psychosis).

Four of the seven had auditory, while two had visual hallucinations. One patient noted spiders crawling up his arm and said that people around him are in 'league with the devil'. One of the seven (14\%) had somatic hallucinations. Only two of the seven had full-range, appropriate affect. One had affect described as constricted, one described as blunted/inappropriate and one described as labile. Two had affect described as unspecified. One had somatic and two had grandiose delusions. Two had a history of disruptive behavior and one of removing intravenous lines. One had a history of self-injury. Additional evidence of abnormal thoughts and behaviors can be found in Tables II and III.

Two of the seven were taking phenothiazines (most often chlorpromazine and fluphenazine). Five of the seven $(71 \%)$ were taking atypical agents (most often olanzapine, quetiapine and risperidone). Four of the seven (57\%) had experienced adverse reactions to their antipsychotic medication.

Symptoms and treatment. Four of the seven inappropriately delayed seeking medical attention. For these four, the mean time from symptom onset to initial treatment was 102 days. None of the four waited $>1$ year. However, the mean wait time from symptom onset to palliative surgery was 130 days. Three of the seven $(43 \%)$ could not give a coherent history. All seven were compliant with their antipsychotic regimen during the course of treatment for $\mathrm{MBC}$, while five were compliant with their MBC therapy.

All seven had a CT, six had a bone scan and three had a chest ultrasound. Six had a chest radiograph, and six had a mammogram during their extent of disease studies.

Five of the seven were offered cytotoxic chemotherapy. Four received initial chemotherapy, while only two completed $\leq 4$ cycles. Five of the seven were offered endocrine therapy, three refused this therapy and only two were compliant. One patient refused tamoxifen, noting that 'I want to see if I am mortal or immortal'. Four of the seven were offered radiation therapy, one refused and three complied with treatment. All seven patients were offered palliative breast surgery, two of whom refused, while five underwent the surgery.

\section{Discussion}

Schizophrenia is a chronic illness of unknown etiology. The phenotype is variable, complex and often terrifying to observers. Individuals with schizophrenia suffer grave abuse worldwide, especially when suffering from a physical illness such as breast carcinoma (19). Recent reports have cited widespread 'neglect, cruel and inhuman treatment, and punishment' of schizophrenic subjects. This is contrary to international law, recommendations from international organizations and statements from various professional groups and government agencies (20). In general, individuals with schizophrenia have difficulty receiving necessary medical treatment (21). The majority of patients have a paranoid component to their illness that decreases their compliance with care. Additionally, limited perception of pain and/or altered expression of the emotional response to pain are well-documented (22). Such patients often have advanced-stage cancer at diagnosis and/ or delayed diagnosis and/or display aggression to healthcare workers (5). Furthermore, a brief gap in antipsychotic medication compliance increases the risk of hospitalization in schizophrenics (23).

This is a population-based rather than a referral-based study. Males are well-represented. A limitation of our study is the small sample size, despite the fact that an extremely large database was used. Furthermore, patients were identified by computer codes for diagnoses of schizophrenia without a means to check their compliance with the diagnostic criteria established by the American Psychiatric Association. It is important to note that schizophrenic patients in the DVA system have direct access to healthcare. The DVA system treats numerous schizophrenic patients since it offers a safety net for veterans, who are often homeless and/or unemployed. A number of schizophrenic patients not eligible for DVA care do not have this access, suggesting that our findings may underestimate the global burden of schizophrenia (24). Another potential confounder is the presence of a strong mental health service component in DVA medical centers, which is not the case in several non-DVA medical centers (25).

There are three main conclusions of our study: i) Since schizophrenic patients with MBC often do not understand the nature of their illness, they frequently do not accept palliative treatment. They often do not comply with therapy, once initiated; ii) often exhibiting behaviors that are detrimental to themselves or others. Detrimental behaviors are likely to be predicted reliably by history alone (Table III). For example, non-adherance to antipsychotic medicine regimens predictably occurs among those who fail to take additional types of medicines (13) and iii) we advise every physician managing any major illness in a patient with schizophrenia to obtain a formal psychiatric consult. There are several reasons for this. The psychiatrist is trained to evaluate whether or not the patient is competent to consent to his/her own medical care and able to judge whether or not the antipsychotic medication regimen is adequate. He/she is also trained to advise the primary caregiver regarding the possibility of the patient's harming themselves or others, and can suggest ways to minimize this hazard. The psychiatrist is often able to marshal resources, such as visits by social workers and admission to shelters, for patients who need them when not hospitalized. During hospitalization, adverse 
events are twice as likely to occur in schizophrenic patients as in non-schizophrenic patients (25). Our chart review provides evidence that, with the appropriate knowledge regarding the difficulties schizophrenia poses on patients and the clinicians who care for them, appropriate full-dose systemic therapy for MBC may be delivered successfully.

The present is believed to be the first study to document the course of treatment in schizophrenic patients with MBC in adequate detail. With this knowledge, physicians can better understand the barriers to providing effective clinical care for this population of patients and take steps to overcome them.

\section{References}

1. American Cancer Society, 2010: Breast Cancer Statistics: Breast cancer overview: how many women get breast cancer. http:// www.cancer.org/Cancer/BreastCancer/OverviewGuide/breastcancer-overview-key-statistics.

2. Stevanovic A, Lee P and Wilcken N: Metastatic breast cancer. Aust Fam Physician 35: 309-312, 2006.

3. Anderson WF, Jatoi I, Tse J and Rosenberg PS: Male breast cancer: a population-based comparison with female breast cancer. J Clin Oncol 28: 232-239, 2010.

4. Nahleh ZA, Srikantiah R, Safa M, et al: Male breast cancer in the veterans affairs population: a comparative analysis. Cancer 109: 1471-1477, 2007.

5. Picchioni MM and Murray RM: Schizophrenia. BMJ 335: 91-95, 2007.

6. McClellan JM, Susser E and King MC: Schizophrenia: a common disease caused by multiple rare alleles. Br J Psychiatry 190: 194-199, 2007.

7. Owen MJ, Williams HJ and O'Donovan MC: Schizophrenia genetics: advancing on two fronts. Curr Opin Genet Dev 19: 266-270, 2009

8. Esslinger C, Walter H, Kirsch P, et al: Neural mechanisms of a genome-wide supported psychosis variant. Science 324: 605, 2009.

9. Tiihonen J, Lönnqvist J, Wahlbeck K, et al: 11-year follow-up of mortality in patients with schizophrenia: a population-based cohort study (FIN11 study). Lancet 374: 620-627, 2009.

10. Akil H, Brenner S, Kandel E, et al: Medicine. The future of psychiatric research: genomes and neural circuits. Science 327: $1580-1581,2010$
11. Fazel S, Långström N, Hjern A, et al: Schizophrenia, substance abuse, and violent crime. JAMA 301: 2016-2023, 2009.

12. Kooyman I, Dean K, Harvey S and Walsh E: Outcomes of public concern in schizophrenia. Br J Psychiatry 50: 29-36, 2007.

13. Velligan DI, Weiden PJ, Sajatovic M, et al: The expert consensus guideline series: adherence problems in patients with serious and persistent mental illness. J Clin Psychiatry 70 (Suppl 4): 1-46, 2009.

14. Hershman DL, Kushi LH, Shao T, et al: Early discontinuation and nonadherence to adjuvant hormonal therapy in a cohort of 8,769 early-stage breast cancer patients. J Clin Oncol 28: 4120-4128, 2010.

15. Sharma A, Ngan S, Nandoskar A, et al: Schizophrenia does not adversely affect the treatment of women with breast cancer: a cohort study. Breast 19: 410-412, 2010.

16. Tran E, Rouillon F, Loze JY, et al: Cancer mortality in patients with schizophrenia: an 11-year prospective cohort study. Cancer 115: 3555-3562, 2009.

17. Congressional Budget Office: The health care system for veterans: an interim report (2006). http://www.cbo.gov/ftpdocs/88xx/ doc8892/MainText.3.1.shtml. Accessed December 31, 2010

18. American Psychiatric Association: Diagnostic and Statistical Manual of Mental Disorders, Text Revision, DSM-IV-TR ${ }^{\circledR}$. 4th edition. American Psychiatric Association, Washington, DC, 2000.

19. Kleinman A: Global mental health: a failure of humanity. Lancet 374: 603-604, 2009.

20. Minas H. Mentally ill patients dying in social shelters in Indonesia. Lancet 374: 592-593, 2009.

21. Bradford DW, Kim MM, Braxton LE, et al: Access to medical care among persons with psychotic and major affective disorders. Psychiatr Serv 59: 847-852, 2008.

22. Talbott JA and Linn L: Reactions of schizophrenics to life-threatening diseases. Psychiatr Q 50: 218-227, 1978.

23. Law MR, Soumerai SB, Ross-Degnan D, et al: A longitudinal study of medication nonadherence and hospitalization risk in schizophrenia. J Clin Psychiatry 69: 47-53, 2008.

24. Abrams TE, Vaughan-Sarrazin M and Rosenthal GE: Influence of psychiatric comorbidity on surgical mortality. Arch Surg 145: 947-953, 2010.

25. Daumit GL, Pronovost PJ, Anthony CB, et al: Adverse events during medical and surgical hospitalizations for persons with schizophrenia. Arch Gen Psychiatry 63: 267-272, 2006. 